\title{
Alpinetin inhibits proliferation and migration of ovarian cancer cells via suppression of STAT3 signaling
}

\author{
XUEZHI ZHAO, XIAOHAN GUO, JUNHUA SHEN and DINGCHAO HUA \\ Department of Gynecology, Women's Hospital, School of Medicine, \\ Zhejiang University, Hangzhou, Zhejiang 310006, P.R. China
}

Received January 11, 2018; Accepted May 23, 2018

DOI: $10.3892 / \mathrm{mmr} .2018 .9420$

\begin{abstract}
Natural bioactive components are increasingly being applied in cancer research. Alpinetin is a type of natural flavonoid primarily derived from Alpinia katsumadai Hayata, which exhibits anti-bacterial and anti-inflammatory properties. Therefore, it may possess anticancer potential and be employed therapeutically for different diseases. The aim of the present study was to investigate the anticancer effects of alpinetin on the SKOV3 ovarian cancer cell line. The effect of alpinetin treatment on SKOV3 cell proliferation, apoptosis, spheroid and colony formation were measured using Cell Counting kit-8, cell apoptosis, 3D spheroid and colony formation assays, respectively. Analysis of the cell cycle was performed using flow cytometry. Western blot analysis was used to determine the protein expression levels of B-cell lymphoma (Bcl)-2, Bcl-2-associated X protein, cleaved caspase-3, cleaved poly (ADP-ribose) polymerase (PARP), cyclin D1, cyclin-dependent kinase (CDK) 4, CDK6, signal transducer and activator of transcription (STAT) 3, phosphorylated (p)-STAT3, c-myc, survivin, tissue inhibitor of metalloproteinase (TIMP)-1, TIMP-2, matrix metalloproteinase (MMP)-2 and MMP-9. In addition, a wound healing assay was used to determine cancer cell migration. The results revealed alpinetin suppressed cell viability and induced apoptosis of SKOV3 cells in a dose- and time-dependent manner, and cells were arrested in the $G_{1}$
\end{abstract}

Correspondence to: Dr Xuezhi Zhao, Department of Gynecology, Women's Hospital, School of Medicine, Zhejiang University, 1 Xueshi Road, Hangzhou, Zhejiang 310006, P.R. China

E-mail: zhaoxz0802@zju.edu.cn

Abbreviations: Bax, Bcl-2-associated X protein; $\mathrm{Bcl}$, B-cell lymphoma; CDK, cyclin-dependent kinase; FACS, fluorescence-activated cell sorting; MMP, matrix metalloproteinase; PARP, poly (ADP-ribose) polymerase; VEGF, vascular endothelial growth factor; PI3K/Akt, phosphatidylinositol-4, 5-bisphosphate 3-kinase/protein kinase B; STAT, signal transducer and activator of transcription; TIMP, tissue inhibitor of metalloproteinase

Key words: alpinetin, ovarian cancer, STAT3, cell migration, colony formation, spheroid phase. Alpinetin treatment upregulated protein expression levels of Bax, cleaved caspase-3 and PARP, and downregulated protein expression levels of Bcl-2, cyclin D1, CDK4 and CDK6. Alpinetin also inhibited cell migration, through increased protein expression levels of TIMP-1 and TIMP-2, and decreased protein expression levels of MMP-2 and MMP-9. Alpinetin also significantly suppressed colony and spheroid formation by SKOV3 cells. In addition, the STAT3 pathway was suppressed as demonstrated by downregulation of p-STAT3 and reduced expression of downstream factors, including c-myc and survivin. Overall, these results indicated that alpinetin may have anticancer effects on human ovarian cancer by inhibiting the STAT3 signaling pathway.

\section{Introduction}

Ovarian cancer is the most fatal gynecological cancer and the fifth most common cause of cancer mortality in women (1-3). Epigenetic and genetic factors can influence the genesis and development of ovarian cancer. Notably, activation of the canonical signal transducer and activator of transcription 3 (STAT3) signaling pathway has a crucial role in inducing tumorigenesis, progression, invasion and metastasis (4-8). Phosphorylated STAT3 translocates to nucleus where it activates its target genes, including B-cell lymphoma 2 (Bcl-2), cyclin D1 and survivin. These genes regulate cell proliferation and apoptosis, thereby mediating cancer initiation, invasion, and progression (4-11). A combination of surgical operation and chemotherapy is currently the most popular therapeutic strategy for patients with ovarian cancer $(1,2)$. The most common chemotherapy regimens for treatment of ovarian cancer employ a combination of carboplatin and paclitaxel. Although up to $80 \%$ of patients respond well to these chemotherapy drugs initially, a majority of patients suffer from recurrence and metastasis due to chemoresistance. The discovery and identification of novel drugs and therapeutic targets in ovarian cancer are required, and botanical products are attractive candidates.

Alpinetin (7-hydroxy-5-methoxyflavanone; $\mathrm{C}_{16} \mathrm{H}_{14} \mathrm{O}_{4}$; Fig. 1A) is a natural flavonoid primarily derived from the roots or seeds of Alpinia katsumadai Hayata that is known to exhibit antibacterial, anti-inflammatory and other important therapeutic properties (12-18). In previous studies, alpinetin was reported to inhibit proliferation of human tumor cells, including lung cancer and gastroenteric cancer cells $(12-16,19)$, indicating the potential 
anticancer properties of this compound. However, the effects of alpinetin on ovarian cancer cells and its underlying mechanisms of action remain largely unknown.

In the present study, the effects of alpinetin on SKOV3 ovarian cancer cells were investigated. The results demonstrated that alpinetin inhibited SKOV3 cell proliferation, led to arrest in the G1/S transition phase and altered expression levels of apoptosis-related protein. In addition, alpinetin suppressed the invasion ability of SKOV3 cells by inhibiting cell migration as well as altering protein expression levels of matrix metalloproteinases (MMPs) and tissue inhibitors of metalloproteinases (TIMPs). Alpinetin also suppressed colony and spheroid formation by SKOV3 cells. It was hypothesized that STAT3 signaling pathway may mediate the anti-ovarian cancer effects of alpinetin.

\section{Materials and methods}

Chemicals and reagents. Alpinetin (>98\% high-performance liquid chromatography purity) was purchased from Chengdu Must Biotechnology Co., Ltd. (Chengdu, China). Alpinetin was dissolved in dimethylsulfoxide (DMSO) to a concentration of $200 \mathrm{mM}$ before use. RPMI-1640 medium, PBS, trypsin/EDTA solution and fetal bovine serum (FBS) were purchased from Gibco (Thermo Fisher Scientific, Inc., Waltham, MA, USA). Cell Counting kit-8 (CCK)-8, bicinchoninic acid (BCA) protein assay kit, Cell Apoptosis Detection kit and Cell Cycle Analysis kit were purchased from Beyotime Institute of Biotechnology (Haimen, China). Antibodies against $\alpha$-tubulin (ab52866; 1:2,000), cyclin D1 (ab134175; 1:10,000), surviving (ab469; 1:2,000), Bcl-2 (ab59348; 1:1,000), Bcl-2-associated X protein (Bax; ab32503; 1:2,000), TIMP-1 (ab61224; 1:500), TIMP-2 (ab180630; 1:500), MMP-2 (ab92536; 1:1,000) and MMP-9 (ab137867; 1:1,000) were obtained from Abcam (Cambridge, UK). Antibodies against cyclin-dependent kinase (CDK)4 (11026-1-AP; 1:500), CDK6 (14052-1-AP; 1:500) and c-myc (10828-1-AP; 1:500) were purchased from Wuhan Sanying Biotechnology (Wuhan, China). Collagen solution $(04902,3 \mathrm{mg} / \mathrm{ml})$ was obtained from STEMCELL Technologies, Inc. (Vancouver, BC, Canada) Antibodies against STAT3 (4904P; 1:1,000), phosphorylated (p)-STAT3 (9145P; 1:1,000), cleaved caspase-3 (9664s; 1:1,000) and cleaved poly (ADP-ribose) polymerase (PARP, 5625S, 1:1,000) were purchased from Cell Signaling Technology, Inc. (Danvers, MA, USA). Fluorescent-labeled secondary antibodies (ABIN2169623/ABIN2169616, 1:10,000) were purchased from LI-COR Biosciences (Lincoln, NE, USA). CellTiter-Glo ${ }^{\circledR}$ 3D Cell Viability Assay kit (G9683) was purchased from Promega Corporation (Madison, WI, USA).

Cell culture. Human SKOV3 ovarian cancer cell line was obtained from EK-Bioscience (Shanghai, China). SKOV3 cells were cultured in RPMI-1640 supplemented with 10\% FBS, penicillin (100 IU) and streptomycin $(100 \mu \mathrm{g} / \mathrm{ml})$ at $37^{\circ} \mathrm{C}$ in a humidified atmosphere containing $5 \% \mathrm{CO}_{2}$.

Cell viability. SKOV3 cells were seeded into 96-well plates (5,000 cells/well) and allowed to adhere overnight before being treated with different concentrations of alpinetin. DMSO was used as vehicle control and RPMI-1640 with 10\% FBS was used as negative control. The maximum concentration of DMSO is $0.25 \%$. Subsequently, cells were cultured for 24 ,

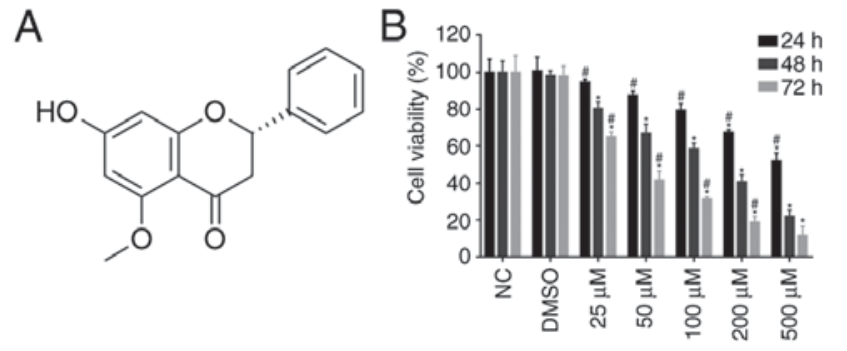

Figure 1. Cell viability of alpinetin-treated SKOV3 cells. (A) Chemical structure of alpinetin. (B) SOKV3 cells were treated with increasing concentrations of alpinetin $(0,25,50,100,200$ and $500 \mu \mathrm{M})$ for 24,48 and $72 \mathrm{~h}$. Cell Counting kit- 8 assay was used to evaluate cell viability. Each experiment was performed three times. ${ }^{*} \mathrm{P}<0.05$ vs. DMSO (vehicle control), ${ }^{\#} \mathrm{P}<0.05$ vs. $48 \mathrm{~h}$. DMSO, dimethylsulfoxide; NC, negative control.

48 and $72 \mathrm{~h}$, and analyzed using the CCK-8 assay according to manufacturer's instructions. Absorbance was measured at $450 \mathrm{~nm}$ using a PerkinElmer Victor3 1420 Multilabel Counter (PerkinElmer, Inc., Waltham, MA, USA). Cell viability (\%) was calculated using the following formula: Cell viability=(average absorbance of samples/average absorbance of controls) x100. All tests were repeated three times.

Apoptosis assay. SKOV3 cells were seeded into 6-well plates $\left(2 \times 10^{5}\right.$ cells/well). Following treatment with different concentrations of alpinetin for $48 \mathrm{~h}$, SKOV3 cells were stained with Annexin V and propidium iodide (PI) using an Apoptosis Detection kit (BB-4101, BestBio, Inc., Shanghai, China) according to the manufacturer's instructions. Briefly, cells were digested from plates, washed twice with cold PBS and resuspended in an Annexin V-fluorescein isothiocyanate (FITC) binding buffer. Annexin V-FITC was added to the cells and mixed gently, and allowed to incubate for $15 \mathrm{~min}$ at $4^{\circ} \mathrm{C}$ in the dark. PI staining solution was then added and incubated for $5 \mathrm{~min}$ at $4^{\circ} \mathrm{C}$ in the dark. Lastly, cells were kept on ice in the dark and immediately analyzed using a FACSCalibur flow cytometer (BD Biosciences, Franklin Lakes, NJ, USA). Cell apoptosis was quantified FlowJo.7.6.1 software (FlowJo LLC, Ashland, OR, USA).

Cell cycle analysis. SKOV3 cells were seeded into 6-well plates $\left(2 \times 10^{5}\right.$ cells/well). After culturing in RPMI-1640 without FBS overnight for synchronization, SKOV3 cells were treated with different concentrations of alpinetin for an additional $48 \mathrm{~h}$ before cells were harvested and fixed in pre-cooled $70 \%$ ethanol at $4^{\circ} \mathrm{C}$ overnight. Fixed cells were washed with PBS and then stained with PI for 30 min at $4^{\circ} \mathrm{C}$ in the dark. Stained cells were acquired using a FACSCalibur flow cytometer (BD Biosciences) and cell cycle was analyzed using FlowJo.7.6.1 software (FlowJo LLC).

Western blot analysis. Following treatment with different concentrations of alpinetin for $48 \mathrm{~h}$, SKOV3 cells were digested from plates and resuspended in ice-cold cell lysis buffer (Beyotime Biotech Co., Ltd.) containing complete ${ }^{\mathrm{TM}}$ mini protease inhibitor cocktail tablet (Roche Diagnostics, Basel, Switzerland). Protein concentration was determined using a BCA kit according to the manufacturer's instructions. Proteins (30 $\mu \mathrm{g} /$ lane) were separated using 10\% SDS-PAGE 

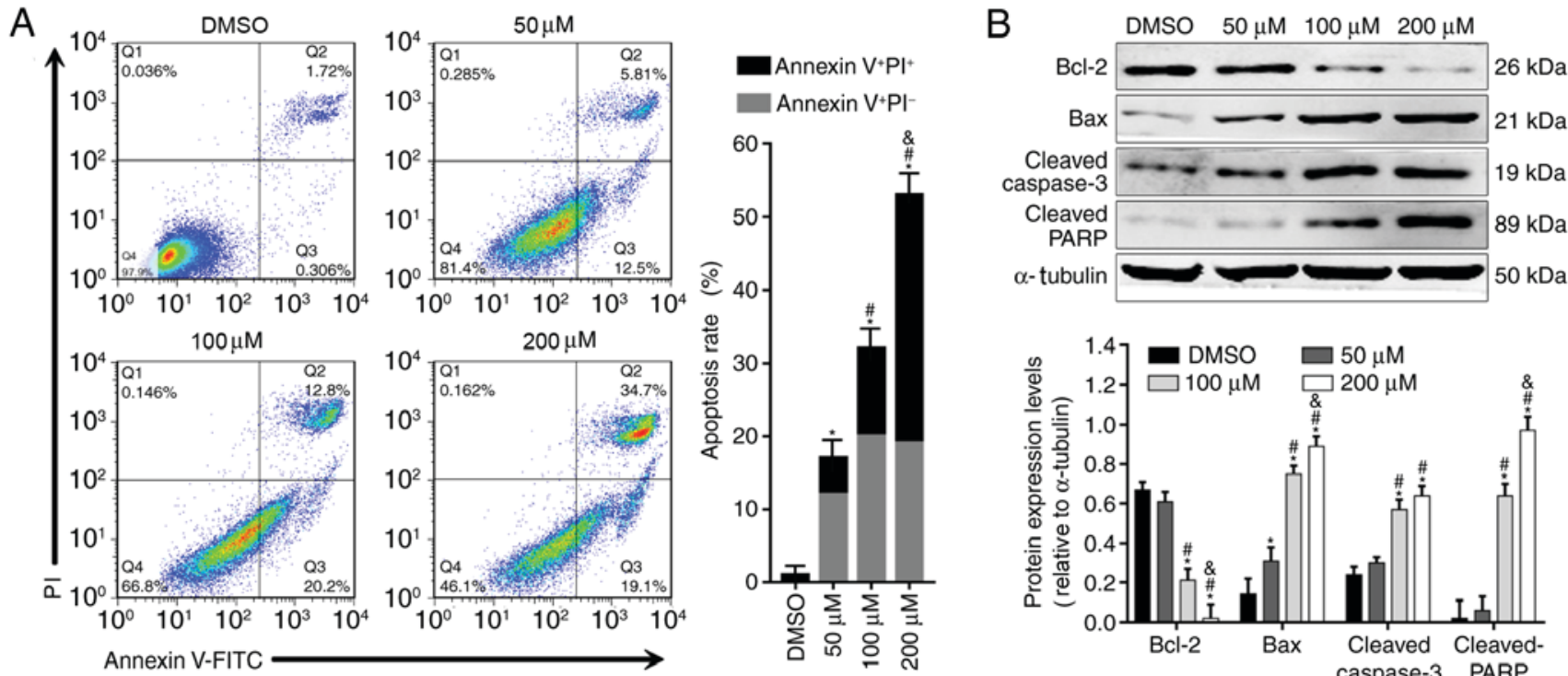

Figure 2. Analysis of apoptosis in alpinetin-treated SKOV3 cells. (A) Apoptosis rates of SKOV3 cells following treatment with increasing concentrations of alpinetin $(50,100$ and $200 \mu \mathrm{M})$ for 48 h. Q1: FITC-PI' ${ }^{+}$Q Q2: FITC ${ }^{+} \mathrm{PI}^{+}$; Q3: FITC-PI-; Q4: FITC'PI- (B) Western blot analysis of apoptosis-related protein expression levels in SKOV3 cells following treatment with increasing concentrations of alpinetin (50, 100 and $200 \mu \mathrm{M})$ for $48 \mathrm{~h}$. Each experiment was performed three times and representative data are shown. ${ }^{*} \mathrm{P}<0.05$ vs. DMSO(vehicle control), ${ }^{\#} \mathrm{P}<0.05$ vs. $50 \mu \mathrm{M},{ }^{\&} \mathrm{P}<0.05$ vs. $100 \mu \mathrm{M}$. Bax, Bcl-2-associated X protein; Bcl-2, B-cell lymphoma-2; DMSO, dimethylsulfoxide; FITC, fluorescein isothiocyanate; PARP, poly (ADP-ribose) polymerase; PI, propidium iodide.

and transferred onto a polyvinylidene fluoride membrane, then blocked with 5\% skimmed milk. Membranes were incubated with primary antibodies overnight at $4^{\circ} \mathrm{C}$. Subsequently, membranes were incubated with secondary antibodies (IRDye700 and IRDye800; goat anti-mouse/rabbit; 1:10,000; LI-COR Biosciences) for $1 \mathrm{~h}$ at $37^{\circ} \mathrm{C}$ and visualized using the Odyssey infrared imaging system (LI-COR Biosciences). The gray value of protein bands were semi-quantified using Image Studio Lite 5.2.5 (LI-COR Biosciences); the protein levels are presented as a ratio of the average gray value of each protein to the average gray value of $\alpha$-tubulin.

Wound healing assay. SKOV3 cells were seeded in 12-well plates $\left(1 \times 10^{5}\right.$ cells $\left./ \mathrm{ml}\right)$ and allowed to adhere overnight. A 'wound' was introduced by scratching the wells using a sharp tip and cells were further incubated for $24 \mathrm{~h}$ with $100 \mu \mathrm{M}$ alpinetin under serum-free conditions. The gap was then imaged under a microscope (CKX31; Olympus Corporation, Tokyo, Japan) and quantified using Image-Pro Plus 5.1 (Media Cybernetics, Inc., Rockville, MD, USA) before and after treatment.

Colony formation assay. SKOV3 cells were seeded into a 96-well plate (200 cells/well) and allowed to adhere overnight. Subsequently, cells were treated with different concentrations of alpinetin for 7 days. The cell culture medium was removed and staining solution $(0.05 \%$ crystal violet, $1 \%$ formaldehyde and $1 \%$ methanol) was added into plate for $20 \mathrm{~min}$. The staining solution was removed, the plate was washed under running water and colonies were counted using an inverted microscope.

$3 D$ spheroid assay and CellTiter-Glo ${ }^{\circledast} 3 D$ cell viability assay. SKOV3 cells were seeded at 5,000 cells/well in ultra-low attachment 96-well plates (Corning Incorporated, Corning, NY, USA) together with collagen ( $5 \mu \mathrm{l} /$ well). Cells were cultured for $24 \mathrm{~h}$ in $37^{\circ} \mathrm{C}$ and spheroid formation was captured using a microscope (IX83; Olympus Corporation). Subsequently, alpinetin was added to wells and DMSO was used as vehicle control. Following 7 days of culture, the spheroids were imaged under a microscope (IX83; Olympus Corporation) before $100 \mu \mathrm{l}$ CellTiter-Glo ${ }^{\circledR}$ 3D reagent was added and allowed to incubate at room temperature in the dark. Luminescence was measured using a Multilabel Counter (PerkinElmer, Inc.).

Statistical analysis. Data are presented as the means \pm standard deviation. The data were subjected to one-way analysis of variance. Differences between two groups were determined using Dunnett's test, and multiple means were compared using Tukey's test. All analyses were conducted using SPSS version 22.0.0.1 (IBM Corp., Armonk, NY, USA). P<0.05 was considered to indicate a statistically significant difference.

\section{Results}

Alpinetin inhibits proliferation of the SKOV3 ovarian cancer cell line. SKOV3 cells were treated with increasing concentrations of alpinetin for 24,48 and $72 \mathrm{~h}$. The CCK- 8 assay was used to investigate whether alpinetin inhibits proliferation of ovarian cancer cells. As shown in Fig. 1B, alpinetin treatment significantly decreased the viability of SKOV3 cells in a dose-and time-dependent manner.

Alpinetin induces cell apoptosis and alters expression levels of apoptosis-associated proteins in SKOV3 cells. Due to the effect of alpinetin on proliferation of SKOV3 cells, it was of interest to perform a cell apoptosis assay to validate the anticancer effect of alpinetin. The results demonstrated that alpinetin induced apoptosis of SKOV3 cells in a dose-dependent manner (Fig. 2A). In addition, treatment of SKOV3 cells with increasing 
A
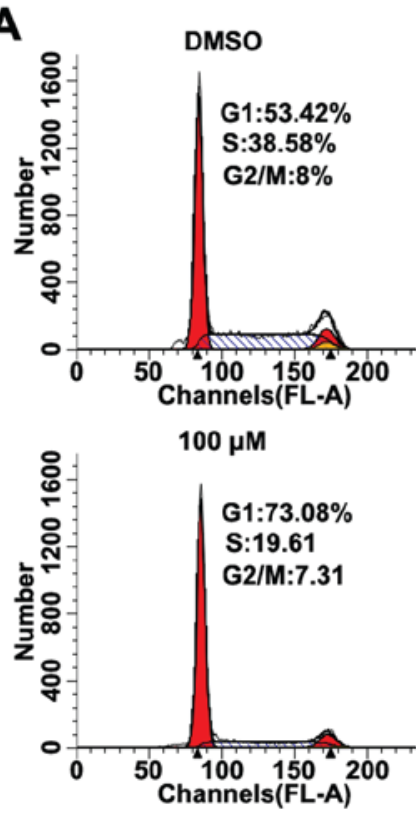
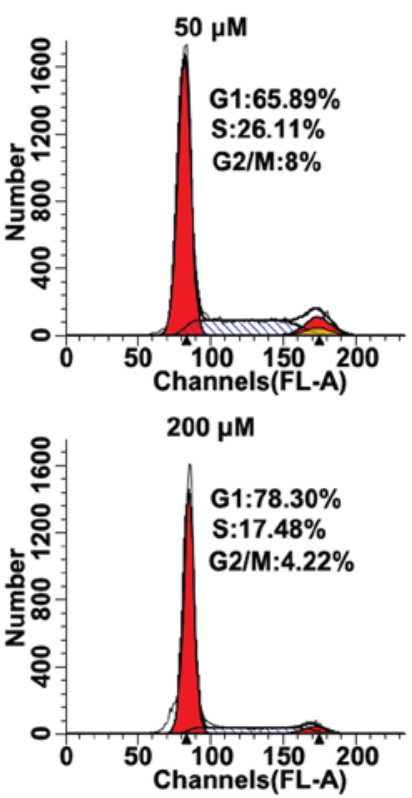

B

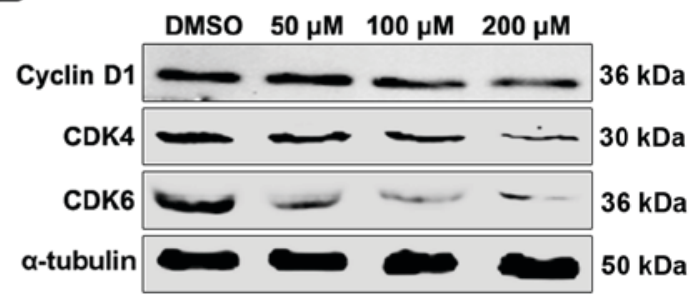

C

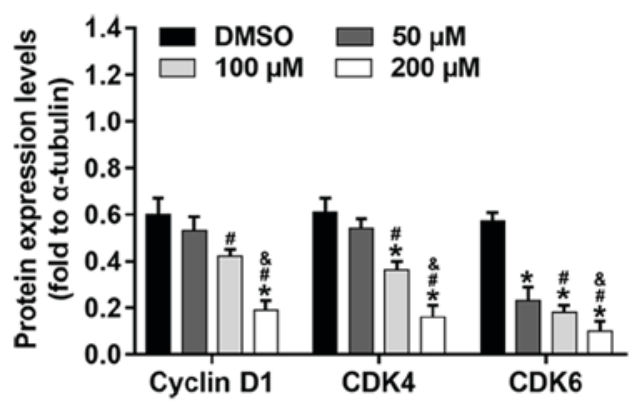

Figure 3. Cell cycle analysis in alpinetin-treated SKOV3 cells. (A) Cell cycle analysis of SKOV3 cells following treatment with increasing concentrations of alpinetin $(50,100$ and $200 \mu \mathrm{M}$ ) for $48 \mathrm{~h}$. (B and C) Western blot analysis of cell cycle-related protein expression levels in SKOV3 cells following treatment with increasing concentrations of alpinetin $(50,100$ and $200 \mu \mathrm{M})$ for $48 \mathrm{~h}$. Each experiment was performed three times and representative data are shown. ${ }^{*} \mathrm{P}<0.05$ vs. DMSO (vehicle control), ${ }^{\#} \mathrm{P}<0.05$ vs. $50 \mu \mathrm{M},{ }^{\&} \mathrm{P}<0.05$ vs. $100 \mu \mathrm{M}$. CDK, cyclin-dependent kinase; DMSO, dimethylsulfoxide.

concentrations of alpinetin significantly downregulated the protein expression levels of Bcl-2, whereas upregulated the protein expression levels of Bax as well as cleaved caspase- 3 and PARP (Fig. 2B).

Alpinetin inhibits G1/S transition and alters expression levels of cell cycle-related proteins. In order to investigate the mechanism underlying alpinetin-mediated inhibition of proliferation of SKOV3 cells, cell cycle was analyzed by flow cytometry and apoptosis-related proteins were detected using western blotting. As shown in Fig. 3A, the number of cells in the $G_{1}$ phase increased whereas the number cells in the $\mathrm{S}$ phase were decreased following alpinetin treatment compared with the DMSO control group. In addition, treatment of SKOV3 cells with increasing concentrations of alpinetin significantly downregulated the protein expression levels of cyclin D1, CDK4 and CDK6 (Fig. 3B and C).

Alpinetin inhibits SKOV3 migration and alters the protein expression levels of TIMPs and MMPs. Migration is one of the key traits of malignant cancer cells. MMPs are relevant for cancer as they are a family of migration promoting factors, whilst TIMPs are inhibitors of MMPs. In the present study, the effects of alpinetin on the migration of SKOV3 cells were evaluated. As shown in Fig. 4A and B, the wound distance was significantly higher in the alpinetin group compared with in the DMSO control group. The results indicated that alpinetin decreased the migratory capacity of SKOV3 cells. In addition, MMP-2 and MMP-9 protein expression levels were significantly decreased in alpinetin-treated cells compared with in control cells (Fig. 4C and D). Conversely, TIMP-1 and TIMP-2 expression levels were increased in the alpinetin-treated groups (Fig. 4C and D).
Alpinetin suppresses colony and spheroid formation of SKOV3 cells. The colony formation ability of SKOV3 cells following alpinetin treatment was also evaluated in the present study and the results demonstrated that treatment with alpinetin significantly suppressed colony formation (Fig. 5A). In addition, the anticancer effects of alpinetin on SKOV3 cells were evaluated in a 3D tumor spheroid culture. Alpinetin decreased the diameter of spheroids compared with the DMSO control group (Fig. 5B) and the cell viability of SKOV3 spheroids was also significantly decreased (Fig. 5C).

STAT3 signaling is involved in the anticancer effects of alpinetin. STAT3 signaling serves an important role in cell proliferation and survival in ovarian cancer. Cyclin D1, Bcl-2 and survivin are STAT3-regulated gene products that are associated with cell proliferation or apoptosis. In order to ascertain whether STAT3 signaling is involved in the anticancer effects of alpinetin on SKOV3 cells, the protein expression levels of STAT3, p-STAT3, c-myc and survivin were detected by western blotting after treatment of cells with different concentrations of alpinetin. The results indicated that alpinetin significantly decreased the phosphorylation of STAT3, and the protein expression levels of c-myc and survivin compared with the DMSO control group (Fig. 6).

\section{Discussion}

Several studies have indicated that alpinetin can inhibit proliferation and induce apoptosis in different cancer cell lines, with numerous signaling pathways, including Notch and phosphatidylinositol-4,5-bisphosphate 3-kinase (PI3K)/protein kinase B (Akt) signaling pathway thought to be involved its anticancer activity $(12,15)$. However, the effects of alpinetin 
A

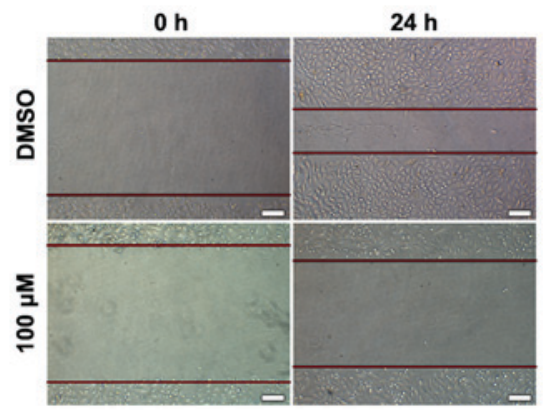

C

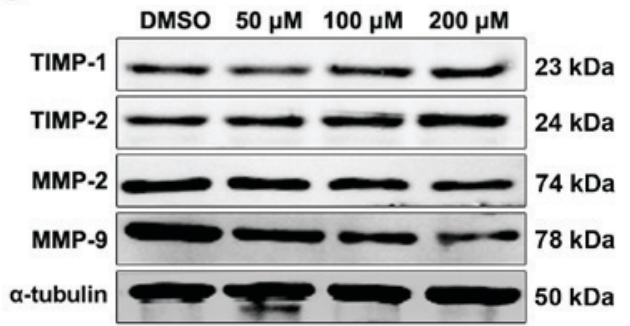

B
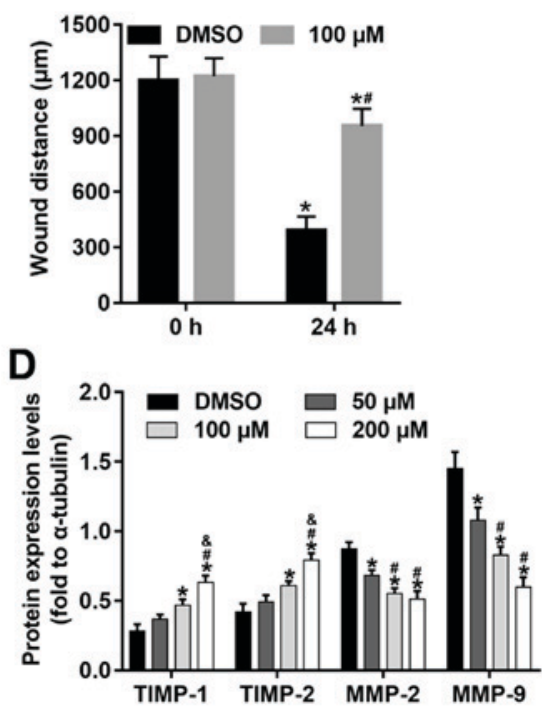

Figure 4. Assessing the effects of alpinetin on SKOV3 cell migration. (A and B) Wound healing assay was performed and wound closure was captured following $24 \mathrm{~h}$. Scale bar, $200 \mu \mathrm{m}$. (C and D) Protein expression levels of TIMP-1, TIMP-2, MMP-2 and MMP-9 were analyzed by western blotting in SKOV3 cells after treatment of alpinetin $(50,100$ and $200 \mu \mathrm{M}$ ) for $48 \mathrm{~h}$. Each experiment was performed three times and representative data are shown. "P $<0.05$ vs. DMSO (vehicle control), ${ }^{\prime \prime} \mathrm{P}<0.05$ vs. $50 \mu \mathrm{M},{ }^{\&} \mathrm{P}<0.05$ vs. $100 \mu \mathrm{M}$. DMSO, dimethylsulfoxide; MMP, matrix metalloproteinase; TIMP, tissue inhibitor of metalloproteinase.

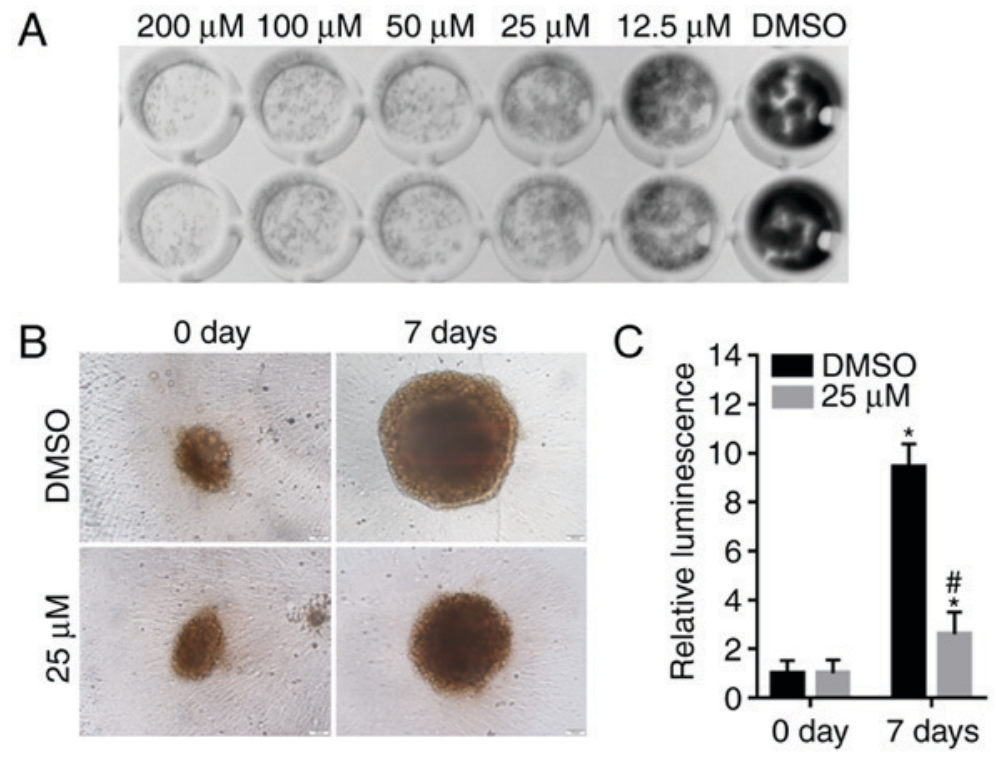

Figure 5. Colony and spheroid formation of alpinetin-treated SKOV3 cells. (A) Colony formation of SKOV3 cells after treatment with alpinetin (12.5, 25, 50, 100 and $200 \mu \mathrm{M}$ ) for 7 days. (B) 3D spheroid assay and spheroid viability was measured following $25 \mu \mathrm{M}$ alpinetin treatment for 7 days and (C) a luminescence assay was used to determine cell viability. Each experiment was performed three times and representative data are shown. ${ }^{~} \mathrm{P}<0.05$ vs. 0 day, ${ }^{"} \mathrm{P}<0.05$ vs. DMSO (vehicle control). DMSO, dimethylsulfoxide.

on ovarian cancer remained poorly understood. The aim of the present study was to investigate the anticancer efficacy of alpinetin and its underlying mechanism of action in ovarian cancer. Alpinetin, a major bioactive component from the roots or seeds of Alpinia katsumadai Hayata, may target SKOV3 ovarian cancer cells in vitro by suppressing proliferation and migration as well as inhibiting the STAT3 signaling pathway.

The results demonstrated that alpinetin inhibited proliferation and induced cell apoptosis of SKOV3 ovarian cancer cells in a dose- and time-dependent manner. Bcl-2 family members are important mediators of cell apoptosis through activating caspase. Caspase- 3 is a crucial member of the caspase family of proteases, whereby cleaved caspase- 3 can cleave PARP and induce apoptosis. The cleaving of caspase-3 and PARP has an important role during cell apoptosis. In the present study, alpinetin significantly decreased the expression levels of anti-apoptotic protein Bax and increased the expression levels of pro-apoptotic proteins Bcl-2, cleaved caspase-3 and PARP in SKOV3 cells. Similarly, Wu et al (12) reported that alpinetin induces cell apoptosis, upregulates the protein expression levels of Bax and cytochrome $c$, and downregulates the protein expression 

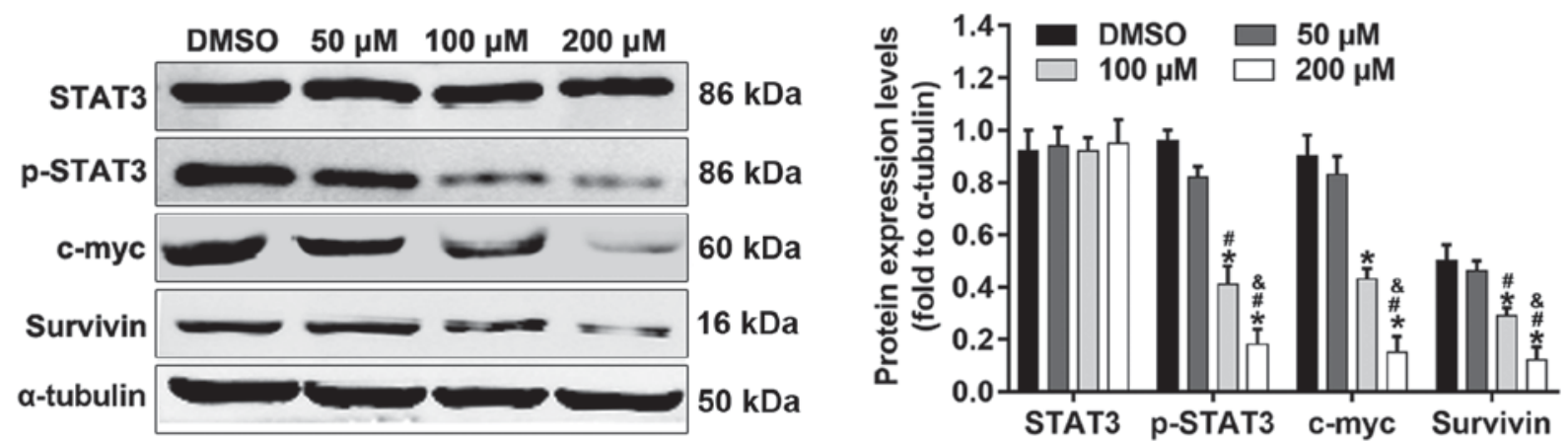

Figure 6. Protein expression levels of STAT3 signaling-related proteins in SKOV3 cells were detected by western blot analysis after treatment with alpinetin $(50,100$ and $200 \mu \mathrm{M})$ for $48 \mathrm{~h}$. Each experiment was performed three times and representative data are shown. *P<0.05 vs. DMSO (vehicle control), ${ }^{\#} \mathrm{P}<0.05$ vs. $50 \mu \mathrm{M},{ }^{\&} \mathrm{P}<0.05$ vs. $100 \mu \mathrm{M}$. DMSO, dimethylsulfoxide; p, phosphorylated; STAT, signal transducer and activator of transcription.

levels of Bcl-2, X-linked inhibitor of apoptosis protein and Bcl-extra large (XL) in A549 lung cancer cells. In addition, cells were arrested in the $\mathrm{G}_{1}$ phase by alpinetin. Cyclin-D1, CDK4 and CDK6 are crucial for the transition from $G_{1}$ to $S$ phase of the cell cycle and their protein expression levels were significantly decreased in SKOV3 cells following alpinetin treatment. Therefore, it may be hypothesized that alpinetin-mediated downregulation of cyclin-D1, CDK4 and CDK6 protein expression leads to $G_{1}$ cell cycle phase arrest. These results indicated that alpinetin inhibited cell proliferation, induced cell apoptosis through $\mathrm{G}_{1}$ phase arrest and altered expression of apoptosis-related proteins.

The migratory ability of SKOV3 cells was also detected after alpinetin treatment. The results revealed that alpinetin significantly decreased the migratory capacity of SKOV3 cells. MMPs and TIMPs are the crucial factors for tumor invasion (7,20), whereby MMPs can promote cancer invasion and TIMPs act as inhibitors of MMPs. In the present study, MMP-2 and MMP-9 expression levels were significantly decreased in alpinetin-treated cells compared with in DMSO control cells. Conversely, TIMP-1 and TIMP-2 expression levels were increased in the alpinetin-treated groups. Similarly, Park et al (21) demonstrated that Alpinia katsumadai suppresses cell migration by inhibiting transglutaminase 2, MMP-2 and MMP-9 expression in HT-1080 cells. Therefore, it can be hypothesized that alpinetin suppresses the migration of ovarian cancer cells via downregulation of MMPs and upregulation of its inhibitors.

The effects of alpinetin on SKOV3 colony and spheroid formation following treatment with alpinetin was also evaluated. The results demonstrated that treatment with alpinetin significantly suppressed the colony formation capacity of SKOV3 ovarian cancer cells and significantly decreased the diameter as well as cell viability of SKOV3 spheroids compared with the DMSO control group. These results further confirm the anticancer effects of alpinetin on ovarian cancer.

Numerous signaling pathways, including STAT3, PI3K/Akt, $\beta$-catenin and Notch signaling pathways can influence tumorigenesis, progression, invasion and metastasis of ovarian cancer $(5,12,15,22)$. Activation of the canonical STAT3 signaling pathway serves a vital role in ovarian cancer. Activated p-STAT3 translocates to nucleus where it activates its downstream target genes, including cyclin-D1 and c-myc, which promote cell cycle progression, as well as survivin, Bcl-2, Bcl-XL and myeloid cell leukemia 1, which suppress apoptosis (5,6,8-11). STAT3 also regulates the expression of MMP-9 and vascular endothelial growth factor, both of which are associated with invasion and angiogenesis (5,6,8-11). In the present study, alpinetin significantly altered the protein expression levels of cyclin-D1, c-myc, survivin, Bcl-2, Bax, TIMP-1, TIMP-2, MMP-2, MMP-9 as well as cleaved caspase-3 and PARP in SKOV3 cells. Therefore, it may be hypothesized that alpinetin exerts its anticancer effects on SKOV3 cells through STAT3 signaling. Subsequently, activation of STAT3 signaling in SKOV3 cells after alpinetin treatment was investigated. The results demonstrated that alpinetin inhibited activation of STAT3 signaling in a dose-dependent manner. Previous studies using A549 lung cancer cells and glioma stem cells revealed that the anticancer effects of alpinetin involved inhibition of PI3K/AKT and Notch signaling $(12,15)$. These results implied that inhibition of ovarian cancer cell proliferation and migration by alpinetin resulted from downregulation of STAT3 signaling, and other signaling pathways may also be involved.

In conclusion, alpinetin represents a novel potential anti-ovarian cancer agent, which has the ability to suppress proliferation, promote cell cycle arrest and inhibit invasion via STAT3 signaling in vitro; however, in vivo studies are required to confirm these results. Overall, alpinetin may be a promising compound for treatment of ovarian cancer.

\section{Acknowledgements}

The authors would like to thank all of the colleagues in Department of Gynecology, Women's Hospital, Zhejiang University, for their technical assistance and helpful suggestions.

\section{Funding}

No funding was received.

\section{Availability of data and materials}

The datasets used and/or analyzed during the current study are available from the corresponding author on reasonable request. 


\section{Authors' contributions}

$\mathrm{XZ}$ was involved in project development, data collection, data analysis and manuscript writing. XG, JS and DH conducted data collection and data analysis.

\section{Ethics approval and consent to participate}

Not applicable.

\section{Patient consent for publication}

Not applicable.

\section{Competing interests}

The authors declare that they have no competing interests.

\section{References}

1. Chien J and Poole EM: Ovarian cancer prevention, screening, and early detection: Report from the 11th biennial ovarian cancer research symposium. Int J Gynecol Cancer 27 (9S Suppl 5): S20-S22, 2017

2. Choi JY, Lee S, Yun SM, Suh DH, Kim K, No JH, Jeong EH and Kim YB: Active hexose correlated compound (AHCC) inhibits the proliferation of ovarian cancer cells by suppressing signal transducer and activator of transcription 3 (STAT3) activation. Nutr Cancer 70: 109-115, 2018

3. Li C, Hong L, Liu C, Min J, Hu M and Guo W: Astragalus polysaccharides increase the sensitivity of SKOV3 cells to cisplatin Arch Gynecol Obstet 297: 381-386, 2017.

4. Chen SH, Murphy DA, Lassoued W, Thurston G, Feldman MD and Lee WM: Activated STAT3 is a mediator and biomarker of VEGF endothelial activation. Cancer Biol Ther 7: 1994-2003, 2008

5. Cai L, Zhang G, Tong X, You Q, An Y, Wang Y, Guo L, Wang T, Zhu D and Zheng J: Growth inhibition of human ovarian cancer cells by blocking STAT3 activation with small interfering RNA. Eur J Obstet Gynecol Reprod Biol 148: 73-80, 2010.

6. Kumar J, Fang H, McCulloch DR, Crowley T and Ward AC: Leptin receptor signaling via Janus kinase 2/Signal transducer and activator of transcription 3 impacts on ovarian cancer cell phenotypes. Oncotarget 8: 93530-93540, 2017.

7. JiaZ,Jia Y,Guo FJ,Chen J,Zhang XW andCui MH:Phosphorylation of STAT3 at Tyr705 regulates MMP-9 production in epithelial ovarian cancer. PLoS One 12: e183622, 2017.

8. Hirano T, Ishihara K and Hibi M: Roles of STAT3 in mediating the cell growth, differentiation and survival signals relayed through the IL-6 family of cytokine receptors. Oncogene 19 2548-2556, 2000
9. Landen CN, Lin YG, Armaiz Pena GN, Das PD, Arevalo JM, Kamat AA, Han LY, Jennings NB, Spannuth WA, Thaker PH, et al: Neuroendocrine modulation of signal transducer and activator of transcription-3 in ovarian cancer. Cancer Res 67: 10389-10396, 2007.

10. Siveen KS, Sikka S, Surana R, Dai X, Zhang J, Kumar AP, Tan BK, Sethi G and Bishayee A: Targeting the STAT3 signaling pathway in cancer: Role of synthetic and natural inhibitors. Biochim Biophys Acta 1845: 136-154, 2014.

11. Harada D, Takigawa N and Kiura K: The role of STAT3 in non-small cell lung cancer. Cancers (Basel) 6: 708-722, 2014

12. Wu L, Yang W, Zhang SN and Lu JB: Alpinetin inhibits lung cancer progression and elevates sensitization drug-resistant lung cancer cells to cis-diammined dichloridoplatium. Drug Des Devel Ther 9: 6119-6127, 2015.

13. Wang Z, Lu W, Li Y and Tang B: Alpinetin promotes Bax translocation, induces apoptosis through the mitochondrial pathway and arrests human gastric cancer cells at the G2/M phase. Mol Med Rep 7: 915-920, 2013.

14. Tang B, Du J, Wang J, Tan G, Gao Z, Wang Z and Wang L: Alpinetin suppresses proliferation of human hepatoma cells by the activation of MKK7 and elevates sensitization to cis-diammined dichloridoplatium. Oncol Rep 27: 1090-1096, 2012.

15. Wang J, Yan Z, Liu X, Che S, Wang C and Yao W: Alpinetin targets glioma stem cells by suppressing Notch pathway. Tumor Biol 37: 9243-9248, 2016.

16. Du J, Tang B, Wang J, Sui H, Jin X, Wang L and Wang Z: Antiproliferative effect of alpinetin in BxPC-3 pancreatic cancer cells. Int J Mol Med 29: 607-612, 2012.

17. Ajmala Shireen P, Abdul Mujeeb VM and Muraleedharan K: Theoretical insights on flavanones as antioxidants and UV filters: A TDDFT and NLMO study. J Photochem Photobiol B 170: 286-294, 2017.

18. Huo M, Chen N, Chi G, Yuan X, Guan S, Li H, Zhong W, Guo W, Soromou LW, Gao R, et al: Traditional medicine alpinetin inhibits the inflammatory response in Raw 264.7 cells and mouse models. Int Immunopharmacol 12: 241-248, 2012.

19. Malami I, Abdul AB, Abdullah R, Kassim NK, Rosli R, Yeap SK, Waziri P, Etti IC and Bello MB: Correction: Crude extracts, flavokawain B and alpinetin compounds from the rhizome of Alpinia mutica induce cell death via UCK2 enzyme inhibition and in turn reduce $18 \mathrm{~S}$ rRNA biosynthesis in HT-29 cells. PLoS One 12: e170233, 2017.

20. Sun X, Lin L, Chen Y, Liu T, Liu R, Wang Z, Mou K, Xu J, Li B and Song H: Nitidine chloride inhibits ovarian cancer cell migration and invasion by suppressing MMP-2/9 production via the ERK signaling pathway. Mol Med Rep 13: 3161-3168, 2016.

21. Park MK, Jo SH, Lee HJ, Kang JH, Kim YR, Kim HJ, Lee EJ, Koh JY, Ahn KO, Jung KC, et al: Novel suppressive effects of cardamonin on the activity and expression of transglutaminase- 2 lead to blocking the migration and invasion of cancer cells. Life Sci 92: 154-160, 2013

22. Tang JM, Min J, Li BS, Hong SS, Liu C, Hu M, Li Y, Yang J and Hong L: Therapeutic effects of punicalagin against ovarian carcinoma cells in association with $\beta$-catenin signaling inhibition. Int J Gynecol Cancer 26: 1557-1563, 2016. 